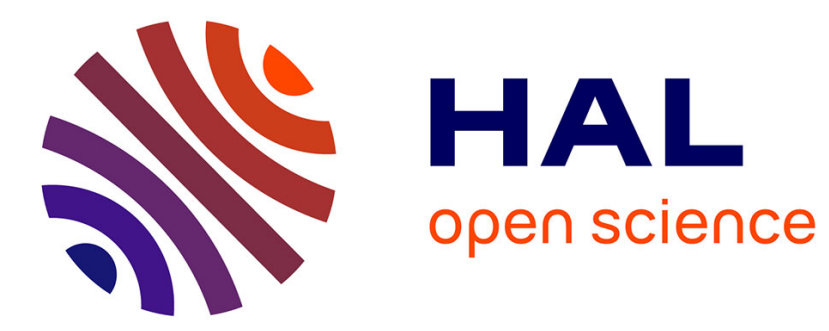

\title{
Discontinuous Lyapunov Functions for Nonasymptotic Stability Analysis
}

Andrey Polyakov

\section{To cite this version:}

Andrey Polyakov. Discontinuous Lyapunov Functions for Nonasymptotic Stability Analysis. IFAC World Congress, Aug 2014, Cape Town, South Africa. hal-00977869

HAL Id: hal-00977869

https://hal.inria.fr/hal-00977869

Submitted on 11 Apr 2014

HAL is a multi-disciplinary open access archive for the deposit and dissemination of scientific research documents, whether they are published or not. The documents may come from teaching and research institutions in France or abroad, or from public or private research centers.
L'archive ouverte pluridisciplinaire HAL, est destinée au dépôt et à la diffusion de documents scientifiques de niveau recherche, publiés ou non, émanant des établissements d'enseignement et de recherche français ou étrangers, des laboratoires publics ou privés. 


\title{
Discontinuous Lyapunov Functions for Nonasymptotic Stability Analysis
}

\author{
A. Polyakov* \\ * Inria Lille-Nord Europe, 40, avenue Halley, Bat.A, Park Plaza 59650 \\ Villeneuve d'Ascq, France (e-mail: andrey.polyakov@inria.fr).
}

\begin{abstract}
The paper presents mathematical tools required for finite-time stability analysis of discontinuous control systems using discontinuous Lyapunov functions. Elements of Filippov theory of differential equations with discontinuous right-hand sides and stability notions are briefly observed. Concepts of generalized derivatives and non-smooth Lyapunov functions are considered. The generalized Lyapunov theorems for stability analysis and convergence time estimation are presented and supported by examples.
\end{abstract}

\section{INTRODUCTION}

During whole history of control theory, a special interest of researchers was focused on systems with relay (switching) control elements (Flugge-Lotz [1953], Tsypkin [1984], Utkin et al. [2009], Boiko [2009]). Such control systems have found applications in many engineering areas. They are simple, effective, cheap and sometimes they have better dynamics than linear systems (Tsypkin [1984]). In practice both input and output of a system may be of a relay type. For example, automobile engine control systems sometimes use $\lambda$ - sensor with almost relay output characteristics, i.e the only sign of a controllable output can be measured (Choi and Hedrick [1996]). In the same time, terristors can be considered as relay "actuators" for some power electronic systems (Utkin et al. [2009]).

Mathematical backgrounds for a rigorous study of relay systems were presented in the beginning of 1960s by the celebrated Filippov's theory of differential equations with discontinuous right-hand sides, see Filippov [1988]. Following this theory, discontinuous differential equations have to be extended to differential inclusions by means of a special procedure. It is worth to stress that Filippov's theory was severely criticized by many authors, see Neimark [1961], Aizerman and Pyatnitskii [1974], Utkin et al. [2009], since it does not describe adequately some discontinuous and relay models. That is why, extensions and specifications of this theory appear rather frequently, see, for example, Bartolini and Zolezzi [1985], Heemels and Weiland [2008].

Analysis of relay systems is frequently related to a specific property, which is called finite-time stability (Roxin [1966], Utkin et al. [2009], Bhat and Bernstein [2000], Orlov [2005], Moulay and Perruquetti [2008]). Indeed, the simplest example of a finite-time stable system is the relay system of the form: $\dot{x}=-\operatorname{sign}[x], x \in \mathbb{R}, x(0)=x_{0}$. Any solution of this system reaches the origin in a finite time $T\left(x_{0}\right)=\left|x_{0}\right|$ and remains there for all later time instants. Sometimes, this conceptually very simple property is hard to proof theoretically. From a practical point of view, it is also important to estimate a time of stabilization (settling time). Both these problems can be tackled by Lyapunov Function Method (Lyapunov [1992], Barbashin
[1970], Bacciotti and Rosier [2005]). However, designing a finite-time Lyapunov function of a rather simple form is a difficult problem for many systems. For concrete relay systems appropriate Lyapunov functions can be nonsmooth (Polyakov and Poznyak [2009b,a]), non-Lipschitz (Moreno and Osorio [2012]) and even discontinuous Polyakov and Poznyak [2012]. Some problems of asymptotic and finitetime stability analysis using Lipschitz continuous Lyapunov functions are studied in, for example, Shevitz and Paden [1994], Bhat and Bernstein [2000], Bacciotti and Ceragioli [2006], Moulay and Perruquetti [2008]. The lower semicontinuous Lyapunov functions for asymptotic stability analysis are discussed in Chellaboina et al. [1999], Clarke et al. [1995], Zhu [2002]

This paper elaborates the non-asymptotic (finite-time) stability analysis of differential inclusions based on discontinuous Lyapunov functions. The main tool to be applied for this purpose is the theory of contingent derivatives (see Zaremba [1934], Natanson [1955], Mordukhovich [2006]).

The paper is organized as follows. The next section presents notations are used in the paper. Then the elements of the Filippov theory differential equations with discontinuous right-hand sides and differential inclusions are discussed. Next the evolution of the stability notion is observed. After then the supporting results about contingent derivatives are studied in order to present a discontinuous Lyapunov function method in the last section.

The following notations are used in the paper $\mathbb{R}$ is the set of real numbers and $\overline{\mathbb{R}}=\mathbb{R} \cup\{-\infty\} \cup\{+\infty\}, \mathbb{R}_{+}=$ $\{x \in \mathbb{R}: x>0\}$ and $\overline{\mathbb{R}}_{+}=\mathbb{R}_{+} \cup\{+\infty\} ; \mathcal{I}$ denotes one of the following intervals: $[a, b],(a, b),[a, b)$ or $(a, b]$, where $a, b \in \bar{R}, a<b$; the set consisting of elements $x_{1}, x_{2}, \ldots, x_{n}$ is denoted by $\left\{x_{1}, x_{2}, \ldots, x_{n}\right\}$; the set of all subsets of a set $M \subseteq \mathbb{R}^{n}$ is denoted by $2^{M}$; the inner product of $x, y \in \mathbb{R}^{n}$ is denoted by $\langle x, y\rangle$ and $\|x\|=\sqrt{\langle x, x\rangle}$; the sign function is defined by

$$
\operatorname{sign}_{\sigma}[\rho]=\left\{\begin{array}{ccc}
1 & \text { if } & \rho>0, \\
-1 & \text { if } & \rho<0, \\
\sigma & \text { if } & \rho=0,
\end{array}\right.
$$

where $\sigma \in \mathbb{R}:-1 \leq \sigma \leq 1$. If $\sigma=0$ we use the notation $\operatorname{sign}[\rho]$; the set-valued modification of the sign function is 
given by

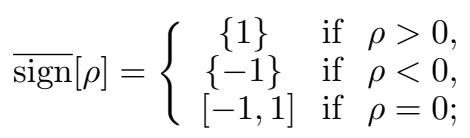

the geometric sum of two sets is denoted by " + ", i.e.

$$
\mathbf{M}_{1} \dot{+} \mathbf{M}_{2}=\bigcup_{x_{1} \in \mathbf{M}_{1}, x_{2} \in \mathbf{M}_{2}}\left\{x_{1}+x_{2}\right\},
$$

where $\mathbf{M}_{1} \subseteq \mathbb{R}^{n}, \mathbf{M}_{2} \subseteq \mathbb{R}^{n}$; the Cartesian product of sets is denoted by $\times ; \overline{\mathcal{B}}(r)=\left\{x \in \mathbb{R}^{n}:\|x\|<r\right\}$ is an open ball of the radius $r \in \mathbb{R}_{+}$with the center at the origin; under introduced notations, $\{y\}+\mathcal{B}(\varepsilon)$ is an open ball of the radius $\varepsilon>0$ with the center at $y \in \mathbb{R}^{n}$; $\operatorname{int}(\Omega)$ is the interior of a set $\Omega \subseteq \mathbb{R}^{n}$, i.e. $x \in \operatorname{int}(\Omega)$ iff $\exists r \in \mathbb{R}_{+}:\{x\} \dot{+} \mathcal{B}(r) \subseteq \Omega$; let $k$ be a given natural number. $\mathbb{C}^{k}(\Omega)$ is the set of continuous functions defined on a set $\Omega \subseteq \mathbb{R}^{n}$, which have continuous partial derivatives of the order $k$ or higher; the set of piecewise continuous functions defined on $\Omega \subset \mathbb{R}^{n}$ is denoted by $\mathbb{C}^{-1}(\Omega)$; if $V(\cdot) \in \mathbb{C}^{1}$ then $\nabla V(x)=\left(\frac{\partial V}{\partial x_{1}}, \ldots, \frac{\partial V}{\partial x_{n}}\right)^{T} ; \mathbb{W}_{\mathcal{I}}^{n}$ is the set of vector-valued, componentwise locally absolutely continuous functions, which map $\mathcal{I}$ to $\mathbb{R}^{n}$.

\section{SYSTEM DESCRIPTION}

Let us consider the system

$$
\dot{x} \in F(t, x), \quad t \in \mathbb{R},
$$

where $x \in \mathbb{R}^{n}$ is a system state, $F: \mathbb{R} \times \mathbb{R}^{n} \rightarrow 2^{\mathbb{R}^{n}}$ is a setvalued mapping, which satisfies the standard assumptions: the set $F(t, x)$ is nonempty, convex and compact for any $(t, x) \in \mathbb{R} \times \mathbb{R}^{n}$; the set-valued mapping $F$ is upper semicontinuous in $\mathbb{R} \times \mathbb{R}^{n}$.

The conventional approach to the regularization of the differential equation with discontinuous right-hand side

$$
\dot{x}=f(t, x), \quad t \in \mathbb{R}, x \in \mathbb{R}^{n}, f \in \mathbb{C}^{-1}
$$

is its extension to the differential inclusion. For instance, the Filippov procedure gives the following construction for the right-hand side of the differential inclusion (4)

$$
F(t, x)=\bigcap_{\delta>0} \bigcap_{\mu(N)=0} \operatorname{co} f(t,\{x\} \dot{+} B(\delta) \backslash N),
$$

where the intersections are taken over all sets $N \subset \mathbb{R}^{n}$ of measure zero $(\mu(N)=0)$ and all $\delta>0$. The Filippov regularization provides the differential inclusions, which satisfy the standard assumptions.

Definition 1. (Filippov [1988], page 50). An absolutely continuous function $x: \mathcal{I} \rightarrow \mathbb{R}^{n}$ defined on some interval or segment $\mathcal{I}$ is called a solution of (5) if it satisfies the differential inclusion (4), (6) almost everywhere on $\mathcal{I}$.

Theorem 1. (Filippov [1988], page 77). Let a set-valued function $F: \mathbf{G} \rightarrow 2^{\mathbb{R}^{n}}$ be defined and upper semi-continuous at each point of the set $\mathbf{G}=\left\{(t, x) \in \mathbb{R}^{n+1}: \mid t-\right.$ $t_{0} \mid \leq a$ and $\left.\left\|x-x_{0}\right\| \leq b\right\}$, where $a, b \in \mathbb{R}_{+}, t_{0} \in \mathbb{R}, x_{0} \in$ $\mathbb{R}^{n}$. Let $F(t, x)$ be nonempty, compact and convex for $(t, x) \in \mathbf{G}$.

If there exists $K>0$ such that $\|y\|<K$ for all $y \in F(t, x)$ and all $(t, x) \in \mathbf{G}$ then there exists at least one absolutely continuous function $x: \mathbb{R} \rightarrow \mathbb{R}^{n}$ defined at least on the segment $\left[t_{0}-\alpha, t_{0}+\alpha\right], \alpha=\min \{a, b / K\}$, such that $x\left(t_{0}\right)=x_{0}$ and the inclusion $\dot{x}(t) \in F(t, x(t))$ holds almost everywhere on $\left[t_{0}-\alpha, t_{0}+\alpha\right]$.
Alternative regularization procedures for discontinuous control systems can be found, for example, in Aizerman and Pyatnitskii [1974], Utkin et al. [2009], Heemels and Weiland [2008].

\section{STABILITY AND CONVERGENCE RATE}

Consider the differential inclusion (4) for $t>t_{0}$ with an initial condition

where $x_{0} \in \mathbb{R}^{n}$ is given.

$$
x\left(t_{0}\right)=x_{0},
$$

Cauchy problem (4), (7) may have non-unique solution for a given $t_{0} \in \mathbb{R}$ and a given $x_{0} \in \mathbb{R}^{n}$. Let us denote the set of all solutions of Cauchy problem (4), (7) by $\Phi\left(t_{0}, x_{0}\right)$ and a solution of (4), (7) by $x\left(t, t_{0}, x_{0}\right) \in \Phi\left(t_{0}, x_{0}\right)$.

Nonuniqueness of solutions implies two types of stability for differential inclusions (4): weak stability (a property holds for a solution) and strong stability (a property holds for all solutions) (see, for example, Filippov [1988]). Weak stability usually is not enough for robust control purposes. This section observes only strong stability properties of the system (4). All conditions presented in definitions below are assumed to be held for all solutions $x\left(t, t_{0}, x_{0}\right) \in$ $\Phi\left(t_{0}, x_{0}\right)$.

\subsection{Lyapunov and asymptotic stability}

The concept of stability introduced in the famous thesis of A.M. Lyapunov (see, its recent translation in Lyapunov [1992]) is the central notion of the modern stability theory. It considers some nominal motion $x^{*}\left(t, t_{0}, x_{0}\right)$ of a dynamic system and studies small perturbations of the initial condition $x_{0}$. If they imply small deviations of perturbed motions from $x^{*}\left(t, t_{0}, x_{0}\right)$ then the nominal motion is called stable. We study different stability forms of the zero solution (or, equivalently, the origin) of the system (4), since making the change of variables $y=x-x^{*}$ it is nothing to transform any problem of stability analysis for some nontrivial solution $x^{*}\left(t, t^{*}, x_{0}^{*}\right)$ to the same problem for the zero solution. Assume that $0 \in F(t, 0), \forall t \in \mathbb{R}$, where $F$ is defined by (4).

Definition 2. (Lyapunov stability). The origin of the system (4) is said to be Lyapunov stable if for $\forall \varepsilon \in \mathbb{R}_{+}$and $\forall t_{0} \in \mathbb{R}$ there exists $\delta=\delta\left(\varepsilon, t_{0}\right) \in \mathbb{R}_{+}$such that :

1 ) any solution $x\left(t, t_{0}, x_{0}\right)$ (with $x_{0} \in \mathcal{B}(\delta)$ ) of Cauchy problem $(4),(7)$ exists for all $\left.t>t_{0} ; 2\right) x\left(t, t_{0}, x_{0}\right) \in \mathcal{B}(\varepsilon)$ for $t>t_{0}$ if $x_{0} \in \mathcal{B}(\delta)$.

If the function $\delta$ does not depend on $t_{0}$ then the origin is called uniformly Lyapunov stable. For instance, if $F(t, x)$ is independent of $t$ (time-invariant case) and the zero solution of (4) is Lyapunov stable, then it is uniformly Lyapunov stable. The origin, which does not satisfy any condition from Definition 2, is called unstable.

Proposition 1. If the origin of (4) is Lyapunov stable then $x(t)=0$ is the unique solution of (4), (7) with $x_{0}=0$.

Definition 3. (Asymptotic attractivity). The origin of the system (4) is said to be asymptotically attractive if for $\forall t_{0} \in \mathbb{R}$ there exists a set $\mathcal{U}\left(t_{0}\right) \subseteq \mathbb{R}^{n}: 0 \in \operatorname{int}\left(\mathcal{U}\left(t_{0}\right)\right)$ :

1) any solution $x\left(t, t_{0}, x_{0}\right)$ (with $\left.x_{0} \in \mathcal{U}\left(t_{0}\right)\right)$ of Cauchy problem (4), (7) exists for $\left.t>t_{0} ; 2\right) \lim _{t \rightarrow+\infty}\left\|x\left(t, t_{0}, x_{0}\right)\right\|=$ 0 . The set $\mathcal{U}\left(t_{0}\right)$ is called attraction domain. 
Definition 4. (Asymptotic stability). The origin of the system (4) is said to be asymptotically stable if it is Lyapunov stable and asymptotically attractive.

If $\mathcal{U}\left(t_{0}\right)=\mathbb{R}^{n}$ then the asymptotically stable (attractive) origin of the system (4) is called globally asymptotically stable (attractive).

Requirement of Lyapunov stability is very important in Definition 4, since even global asymptotic attractivity does not imply Lyapunov stability (see, for example, Vinograd [1957], page 433 or Hahn [1967], page 191).

\subsection{Finite-time Stability}

Introduce the functional $T_{0}: \mathbb{W}_{\left[t_{0},+\infty\right)}^{n} \rightarrow \overline{\mathbb{R}}_{+} \cup\{0\}$ by the following formula $T_{0}(y(\cdot))=\inf _{\tau \geq t_{0}: y(\tau)=0} \tau$. If $y(\tau) \neq 0$ for all $t \in\left[t_{0},+\infty\right)$ then $T_{0}(y(\cdot))=+\infty$.

The settling-time function of the system (4) let us define by

$$
T\left(t_{0}, x_{0}\right)=\sup _{x\left(t, t_{0}, x_{0}\right) \in \Phi\left(t_{0}, x_{0}\right)} T_{0}\left(x\left(t, t_{0}, x_{0}\right)\right)-t_{0},
$$

where $\Phi\left(t_{0}, x_{0}\right)$ is the set of all solutions of the Cauchy problem (4), (7).

Definition 5. (Finite-time attractivity). The origin of the system (4) is said to be finite-time attractive if for $\forall t_{0} \in \mathbb{R}$ there exists a set $\mathcal{V}\left(t_{0}\right) \subseteq \mathbb{R}^{n}: 0 \in \operatorname{int}\left(\mathcal{V}\left(t_{0}\right)\right)$ :

1) any solution $x\left(t, t_{0}, x_{0}\right)$ (with $x_{0} \in \mathcal{V}\left(t_{0}\right)$ ) of Cauchy problem (4), (7) exists for all $t>t_{0}$; 2) $T\left(t_{0}, x_{0}\right)<+\infty$ for $x_{0} \in \mathcal{V}\left(t_{0}\right)$ and for $t_{0} \in R$. The set $\mathcal{V}\left(t_{0}\right)$ is called finite-time attraction domain.

It is worth to stress that the presented finite-time attractivity property, introduced originally in Bhat and Bernstein [2000], is "weaker" than asymptotic attractivity. However, it is important for many control applications. For example, antimissile control problem has to be studied only on a finite interval of time, since there is nothing to control after missile explosion. In practice, Lyapunov stability is additionally required in order to guarantee a robustness of a control system.

Definition 6. (Finite-time stability (Roxin [1966])). The origin of the system (4) is said to be finite-time stable if it is Lyapunov stable and finite-time attractive.

If $\mathcal{V}\left(t_{0}\right)=\mathbb{R}^{n}$ then the origin of (4) is called globally finitetime stable.

Example 1. Consider the system

$$
\dot{x} \in-\frac{2}{\sqrt{\pi}} \cdot \overline{\operatorname{sign}}[x] \dot{+}\{|2 t x|\}, \quad t>t_{0}, \quad x \in \mathbb{R},
$$

where $t_{0} \in \mathbb{R}$. It can be shown that the origin of this system is finite-time attractive with an attraction domain $\mathcal{V}\left(t_{0}\right)=$ $\mathcal{B}\left(e^{t_{0}^{2}}\left(1-\operatorname{erf}\left(\left|t_{0}\right|\right)\right)\right)$, where $\operatorname{erf}(z)=\frac{2}{\sqrt{\pi}} \int_{0}^{z} e^{-\tau^{2}} d \tau, z \in$ $\mathbb{R}$ is the so-called Gauss error function. Moreover, the origin of the considered system is Lyapunov stable (for $\forall \varepsilon>0$ and for $\forall t_{0} \in \mathbb{R}$ we can select $\delta=\delta\left(t_{0}\right)=$ $\left.\min \left\{\varepsilon, e^{t_{0}^{2}}\left(1-\operatorname{erf}\left(\left|t_{0}\right|\right)\right)\right\}\right)$, so it is finite-time stable. In particular, for $t_{0}>0$ the settling-time function has the form $T\left(t_{0}, x_{0}\right)=\operatorname{erf}^{-1}\left(\left|x_{0}\right| e^{-t_{0}^{2}}+\operatorname{erf}\left(t_{0}\right)\right)-t_{0}$, where $\operatorname{erf}^{-1}(\cdot)$ denotes the inverse function to $\operatorname{erf}(\cdot)$.
The proposition 1 implies the following claim.

Proposition 2. (Bhat and Bernstein [2000]). If the origin of the system (4) is finite-time stable then it is asymptotically stable and $x\left(t, t_{0}, x_{0}\right)=0$ for $t>t_{0}+T_{0}\left(t_{0}, x_{0}\right)$.

\section{GENERALIZED DERIVATIVES}

The celebrated Second Lyapunov Method is founded on the so-called energetic approach to stability analysis. It considers any positive definite function as an possible energetic characteristic ("energy") of a dynamic system and studies evolution of this "energy" in time. If a dynamic system has an energetic function, which is decreasing (strictly decreasing) along any trajectory of the system, then this system has a stability property and the corresponding energetic function is called Lyapunov function.

For example, to analyze asymptotic stability of the origin of the system

$$
\dot{x}=f(t, x), \quad f \in \mathbb{C}\left(\mathbb{R}^{n+1}\right), \quad t \in \mathbb{R}_{+}, x \in \mathbb{R}^{n}
$$

it is sufficient to find a continuous positive definite function $V(\cdot)$ such that for any solution $x(t)$ of the system (10) the function $V(x(t))$ is decreasing and tending to zero for $t \rightarrow+\infty$. The existence of such function guarantees asymptotic stability of the origin of the system (10) due to Zubov's theorem (see, Poznyak [2008]).

If the function $V(x)$ is continuously differentiable then the required monotonicity property can be rewritten in the form of the classical condition Lyapunov [1992]:

$$
\dot{V}(x(t))=\nabla^{T} V(x) f(t, x)<0 .
$$

The inequality (11) is very usable, since it does not require knowing the solutions of (10) in order to check the asymptotic stability. From the practical point of view, it is important to represent monotonicity conditions in the form of differential or algebraic inequalities like (11).

Finite-time stability analysis of relay systems is frequently based on non-smooth or even discontinuous Lyapunov functions Roxin [1966], Polyakov and Poznyak [2009b], Moreno and Osorio [2012], which require consideration of generalized derivatives and generalized gradients in order to verify stability conditions. This section presents all necessary backgrounds for the corresponding non-smooth analysis.

\subsection{Derivative Numbers and Monotonicity}

Let $\mathcal{I}$ be one of the following intervals: $[a, b],(a, b),[a, b)$ or $(a, b]$, where $a, b \in \bar{R}, a<b$.

Recall that the function $\varphi: \mathbb{R} \rightarrow \mathbb{R}$ is called decreasing on $\mathcal{I}$ iff $\forall t_{1}, t_{2} \in \mathcal{I}: t_{1} \leq t_{2} \Rightarrow \varphi\left(t_{1}\right) \geq \varphi\left(t_{2}\right)$.

Let $\mathbb{K}$ be a set of all sequences of real numbers converging to zero, i.e. $\left\{h_{n}\right\} \in \mathbb{K} \quad \Leftrightarrow \quad h_{n} \rightarrow 0, h_{n} \neq 0$.

Let a real-valued function $\varphi: \mathbb{R} \rightarrow \mathbb{R}$ be defined on $\mathcal{I}$.

Definition \%. (Natanson [1955], page 207). A number $D_{\left\{h_{n}\right\}} \varphi(t)=\lim _{n \rightarrow+\infty} \frac{\varphi\left(t+h_{n}\right)-\varphi(t)}{h_{n}},\left\{h_{n}\right\} \in \mathbb{K}: t+h_{n} \in \mathcal{I}$ is called derivative number of the function $\varphi$ at a point $t \in \mathcal{I}$, if finite or infinite limit exists.

The set of all derivative numbers of the function $\varphi$ at the point $t \in \mathcal{I}$ is called contingent derivative:

$$
D_{\mathbb{K}} \varphi(t)=\bigcup_{\left\{h_{n}\right\} \in \mathbb{K}}\left\{D_{\left\{h_{n}\right\}} \varphi(t)\right\} \subseteq \overline{\mathbb{R}} .
$$


A contingent derivative of a vector-valued function $\varphi$ : $\mathbb{R} \rightarrow \mathbb{R}^{n}$ can be defined in the same way. If a function $\varphi(t)$ is differentiable at a point $t \in \mathcal{I}$ then $D_{\mathbb{K}} \varphi(t)=\{\dot{\varphi}(t)\}$.

Lemma 1. (Natanson [1955], page 208). If a function $\varphi$ : $\mathbb{R} \rightarrow \mathbb{R}$ is defined on $\mathcal{I}$ then

1) a set $D_{\mathbb{K}} \varphi(t) \subseteq \overline{\mathbb{R}}$ is nonempty for any $t \in \mathcal{I}$;

2) for any $t \in \overline{\mathcal{I}}$ and for any sequence $\left\{h_{n}\right\} \in \mathbb{K}: t+$ $\left\{h_{n}\right\} \in \mathcal{I}$ there exists a subsequence $\left\{h_{n^{\prime}}\right\} \subseteq\left\{h_{n}\right\}$ such that finite or infinite derivative number $D_{\left\{h_{n^{\prime}}\right\}} \varphi(t)$ exists.

Lemma 1 remains true for a vector-valued function $\varphi$ : $\mathbb{R} \rightarrow \mathbb{R}^{n}$. Inequalities $y<0, y \leq 0, y>0, y \geq 0$ for $y \in \mathbb{R}^{n}$ are understood in a componentwise sense. If for $\forall y \in D_{\mathbb{K}} \varphi(t)$ we have $y<0$ then we write $D_{\mathbb{K}} \varphi(t)<0$. Other ordering relations $\leq,>, \geq$ for contingent derivatives are interpreted analogously.

The contingent derivative also helps to prove monotonicity of a non-differentiable function.

Lemma 2. (Natanson [1955], page 266). If a function $\varphi$ : $\mathbb{R} \rightarrow \mathbb{R}$ is defined on $\mathcal{I}$ and the inequality $D_{\mathbb{K}} \varphi(t) \leq 0$ holds for all $t \in \mathcal{I}$, then $\varphi(t)$ is decreasing function on $\mathcal{I}$ and differentiable almost everywhere on $\mathcal{I}$.

Lemma 2 require neither the continuity of the function $\varphi(t)$ nor the finiteness of its derivative numbers. It gives a background for the discontinuous Lyapunov function method.

Example 2. The function $\varphi(t)=-t-\operatorname{sign}_{\sigma}[t]$ has a negative contingent derivative for all $t \in \mathbb{R}$ and for any $\sigma \in[-1,1]$, where the function $\operatorname{sign}_{\sigma}$ is defined by (1). Indeed, $D_{\mathbb{K}} \varphi(t)=\{-1\}$ for $t \neq 0, D_{\mathbb{K}} \varphi(0)=\{-\infty\}$ if $\sigma \in(-1,1)$ and $D_{\mathbb{K}} \varphi(0)=\{-\infty,-1\}$ if $\sigma \in\{-1,1\}$.

The next lemma simplifies the monotonicity analysis of nonnegative functions.

Lemma 3. If 1 ) the function $\varphi: \mathbb{R} \rightarrow \mathbb{R}$ is nonnegative on $\mathcal{I}$; 2) the inequality $D_{\mathbb{K}} \varphi(t) \leq 0$ holds for $t \in \mathcal{I}: \varphi(t) \neq 0$; 3 ) the function $\varphi(t)$ is continuous at any $t \in \mathcal{I}: \varphi(t)=0$; then $\varphi(t)$ is decreasing function on $\mathcal{I}$ and differentiable almost everywhere on $\mathcal{I}$.

The lemma of Wazewski given below presents the relation between solutions of a differential inclusion (4) and its contingent derivatives.

Lemma 4. (Filippov [1988], page 70). Let a set-valued function $F: \mathbb{R}^{n+1} \rightarrow 2^{\mathbb{R}^{n}}$ be defined, upper-semicontinuous on a closed nonempty set $\Omega \in \mathbb{R}^{n+1}$ and the set $F(t, x)$ be nonempty, compact and convex for all $(t, x) \in \Omega$. Let an absolutely continuous function $x: \mathbb{R} \rightarrow \mathbb{R}^{n}$ be defined on $\mathcal{I}$ and $(t, x(t)) \in \Omega$ if $t \in \mathcal{I}$. Then

$$
\left.\begin{array}{c}
\dot{x}(t) \in F(t, x(t)) \\
\text { almost everywhere on } \mathcal{I}
\end{array}\right\} \Leftrightarrow \begin{gathered}
D_{\mathbb{K}} x(t) \subseteq F(t, x(t)) \\
\text { everywhere on } \mathcal{I} .
\end{gathered}
$$

\subsection{Generalized directional derivatives}

Stability analysis based on Lyapunov functions requires calculation of derivatives of positive definite functions along trajectories of a dynamic system. If Lyapunov function is non-differentiable, a concept of generalized directional derivatives (see, for example, Clarke [1990]) can be used for this analysis. This survey introduces generalized directional derivatives by analogy with contingent derivatives for scalar functions.
Let $\mathbb{M}(d)$ be a set of all sequences of real vectors converging to $d \in \mathbb{R}^{n}$, i.e. $\left\{v_{n}\right\} \in \mathbb{M}(d) \quad \Leftrightarrow \quad v_{n} \rightarrow d, v_{n} \in \mathbb{R}^{n}$.

Let a function $V: \mathbb{R}^{n} \rightarrow \mathbb{R}$ be defined on an open nonempty set $\Omega \subseteq \mathbb{R}^{n}$ and $d \in \mathbb{R}^{n}$.

Definition 8. A number

$$
\begin{gathered}
D_{\left\{h_{n}\right\},\left\{v_{n}\right\}} V(x, d)=\lim _{n \rightarrow+\infty} \frac{V\left(x+h_{n} v_{n}\right)-V(x)}{h_{n}}, \\
\left\{h_{n}\right\} \in \mathbb{K},\left\{v_{n}\right\} \in \mathbb{M}(d): x+h_{n} v_{n} \in \Omega
\end{gathered}
$$

is called directional derivative number of the function $V(x)$ at the point $x \in \Omega$ on the direction $d \in \mathbb{R}^{n}$, if finite or infinite limit exists.

The set of all directional derivative numbers of the function $V(x)$ at the point $x \in \Omega$ on the direction $d \in \mathbb{R}^{n}$ is called directional contingent derivative:

$$
D_{\mathbb{K}, \mathbb{M}(d)} V(x)=\bigcup_{\left\{h_{n}\right\} \in \mathbb{K},\left\{v_{n}\right\} \in \mathbb{M}(d)}\left\{D_{\left\{h_{n}\right\},\left\{v_{n}\right\}} V(x, d)\right\} .
$$

Similarly to Lemma 1 , if $x \in \Omega$ then the set $D_{\mathbb{K}, \mathbb{M}(d)} V(x)$ is nonempty for any function $V$ defined on an open nonempty set $\Omega \subseteq \mathbb{R}^{n}$ and any $d \in \mathbb{R}^{n}$. The next lemma gives a chain rule for the introduced contingent derivative.

Lemma 5. Let a function $V: \mathbb{R}^{n} \rightarrow \mathbb{R}$ be defined on an open nonempty set $\Omega \subseteq \mathbb{R}^{n}$ and a function $x: \mathbb{R} \rightarrow \mathbb{R}^{n}$ be defined on $\mathcal{I}$, such that $x(t) \in \Omega$ if $t \in \mathcal{I}$ and the contingent derivative $D_{\mathbb{K}} x(t) \subseteq \mathbb{R}^{n}$ is bounded for all $t \in \mathcal{I}$. Then the inclusion $D_{\mathbb{K}} V(x(t)) \subseteq \bigcup_{d \in D_{\mathbb{K}} x(t)} D_{\mathbb{K}, \mathbb{M}(d)} V(x)$ holds $\forall t \in \mathcal{I}$.

This lemma together with Lemmas 4 and 3 imply the corollary, which is useful for Lyapunov analysis.

Corollary 1. Let a set-valued function $F: \mathbb{R}^{n+1} \rightarrow 2^{\mathbb{R}^{n}}$ be defined and upper-semicontinuous on $\mathcal{I} \times \Omega$ and a set $F(t, x)$ be nonempty, compact and convex for any $(t, x) \in \mathcal{I} \times \Omega$, where $\Omega \subseteq \mathbb{R}^{n}$ is an open nonempty set. Let $x\left(t, t_{0}, x_{0}\right)$ be an arbitrary solution of Cauchy problem (4), (7) defined on $\left[t_{0}, t_{0}+\alpha\right)$, where $t_{0} \in \mathcal{I}, x_{0} \in \Omega$ and $\alpha \in \mathbb{R}_{+}$. Let a function $V: \mathbb{R}^{n} \rightarrow \mathbb{R}$ be nonnegative on $\Omega$. If the inequality $D_{F(t, x)} V(x) \leq 0$ holds for every $t \in \mathcal{I}$ and every $x \in \Omega: V(x) \neq 0$ then the function of time $V\left(x\left(t, t_{0}, x_{0}\right)\right)$ is decreasing on $\left[t_{0}, t_{0}+\alpha\right)$, where

$$
D_{F(t, x)} V(x)=\bigcup_{d \in F(t, x)} D_{\mathbb{K}, \mathbb{M}(d)} V(x)
$$

\section{DISCONTINUOUS LYAPUNOV FUNCTION} METHOD FOR FINITE-TIME STABILITY ANALYSIS

Lyapunov function method is a very effective tool for analysis and design of both linear and nonlinear control systems Bacciotti and Rosier [2005]. Initially, the method was presented for "unrated" (Lyapunov and asymptotic) stability analysis Lyapunov [1992]. A development of control theory had required to study a convergence rate together with a stability properties of a control system. This section presents the discontinuous Lyapunov function method for finite-time stability analysis. Let us refund initially the Zubov's theorems about Lyapunov and asymptotic stability using the contingent derivative approach.

\subsection{Analysis of Lyapunov and asymptotic stability}

Recall that a continuous function $W: \mathbb{R}^{n} \rightarrow \mathbb{R}$ defined on $\mathbb{R}^{n}$ is said to be positive definite iff $W(0)=0$ and $W(x)>0$ for $x \in \mathbb{R}^{n} \backslash\{0\}$. 
Definition 9. A function $V: \mathbb{R}^{n} \rightarrow \mathbb{R}$ is said to be proper on an open nonempty set $\Omega \subseteq \mathbb{R}^{n}: 0 \in \operatorname{int}(\Omega)$ iff

1 ) it is defined on $\Omega$ and continuous at the origin;

2 ) there exists a continuous positive definite function $\underline{V}$ :

$\mathbb{R}^{n} \rightarrow \mathbb{R}$ such that $\underline{V}(x) \leq V(x)$ for $x \in \Omega$.

A positive definite function $W: \mathbb{R}^{n} \rightarrow \mathbb{R}$ is called radially unbounded if $W(x) \rightarrow+\infty$ for $\|x\| \rightarrow+\infty$.

Definition 10. A function $V: \mathbb{R}^{n} \rightarrow \mathbb{R}$ is said to be globally proper iff it is proper on $\mathbb{R}^{n}$ and the positive definite function $\underline{V}: \mathbb{R}^{n} \rightarrow \mathbb{R}$ is radially unbounded.

If $V$ is continuous on $\Omega$, then $\underline{V}(x)=V(x)$ for $x \in \Omega$ and Definition 10 corresponds to the usual notion of proper positive definite function (see Clarke et al. [1998]).

For a given number $r \in \mathbb{R}$ and a given positive definite function $W: \mathbb{R}^{n} \rightarrow \mathbb{R}$ defined on $\Omega$ let us introduce the set $\Pi(W, r)=\{x \in \Omega: W(x)<r\}$, which is called the level set of the function $W$.

Theorems on Lyapunov and asymptotic stability given below are obtained by a combination of Zubov's theorems (see, for example, Poznyak [2008], pages 566-568) with Corollary 1.

Theorem 2. Let a function $V: \mathbb{R}^{n} \rightarrow \mathbb{R}$ be proper on an open nonempty set $\Omega \subseteq \mathbb{R}^{n}: 0 \in \operatorname{int}(\Omega)$ and

$$
D_{F(t, x)} V(x) \leq 0 \quad \text { for } \quad t \in \mathbb{R} \text { and } x \in \Omega \backslash\{0\} .
$$

Then the origin of the system (4) is Lyapunov stable.

Asymptotic stability requires analysis of an attraction set. Lyapunov function approach may provide an estimate of this set.

Theorem 3. Let a function $V: \mathbb{R}^{n} \rightarrow \mathbb{R}$ be proper on an open nonempty set $\Omega \subseteq \mathbb{R}^{n}: 0 \in \operatorname{int}(\Omega)$, a function $W: \mathbb{R}^{n} \rightarrow \mathbb{R}$ be a continuous positive definite and

$$
D_{F(t, x)} V(x) \leq-W(x) \quad \text { for } \quad t \in \mathbb{R} \text { and } x \in \Omega \backslash\{0\} .
$$

Then the origin of the system (4) is asymptotically stable with an attraction domain

$$
\begin{gathered}
\mathcal{U}=\Pi(V, \lambda(h)) \cap \mathcal{B}(h), \\
\text { where } \lambda(h)=\inf _{x \in \mathbb{R}^{n}:\|x\|=h} \underline{V}(x) \text { and } h=\sup _{r \in \mathbb{R}_{+}: \mathcal{B}(r) \subseteq \Omega} r .
\end{gathered}
$$

If $V$ is globally proper and $\Omega=\mathbb{R}^{n}$ then the origin of the system (4) is globally asymptotically stable $\left(\mathcal{U}=\mathbb{R}^{n}\right)$.

If $V$ is locally bounded on $\Omega$ then the origin of the system (4) is uniformly asymptotically stable.

The presented theorems shows that discontinuous and non-Lipschitz Lyapunov functions can also be used for stability analysis using the theory of contingent derivatives.

The following important theorem declares that a smooth Lyapunov function always exists for a time-invariant asymptotically stable differential inclusion (4).

Theorem 4. (Clarke et al. [1998], Theorem 1.2). Let a set-valued function $F: R^{n} \rightarrow \mathbb{R}^{n}$ be defined and uppersemicontinuous in $\mathbb{R}^{n}$. Let $F(x)$ be nonempty, compact and convex for any $x \in \mathbb{R}^{n}$. If the origin of the system $\dot{x} \in F(x)$ is globally uniformly asymptotically stable iff there exists a globally proper function $V(\cdot) \in \mathbb{C}^{\infty}\left(\mathbb{R}^{n}\right)$ and a function $W(\cdot) \in \mathbb{C}^{\infty}\left(\mathbb{R}^{n}\right): W(x)>0$ for $x \neq 0$ such that $\max _{y \in F(x)} \nabla^{T} V(x) y \leq-W(x), \quad x \in \mathbb{R}^{n} \backslash\{0\}$.
However, the practice shows that designing of a Lyapunov function for nonlinear and/or discontinuous system is a nontrivial problem even for a two dimensional case. Frequently, in order to analyze stability of a sliding mode control system it is simpler to design initially a non-smooth Lyapunov function (see, for example, Utkin et al. [2009], Polyakov and Poznyak [2012], Moreno and Osorio [2012]) and after then to use some technique for its smoothing, see, for example, Clarke et al. [1998].

\subsection{Lyapunov analysis of finite-time stability}

Analysis of finite-time stability using the Lyapunov function method allows us to estimate of a settling time $a$ priori. The proof of the next theorem follows the ideas introduced in Roxin [1966], Polyakov and Poznyak [2012]. Theorem 5. Let a function $V: \mathbb{R}^{n} \rightarrow \mathbb{R}$ be proper on an open nonempty set $\Omega \subseteq \mathbb{R}^{n}: 0 \in \operatorname{int}(\Omega)$ and

$$
D_{F(t, x)} V(x) \leq-q \quad \text { for } \quad t \in \mathbb{R} \text { and } x \in \Omega \backslash\{0\},
$$

for some $q \in \mathbb{R}^{+}$. Then the origin of the system (4) is finite-time stable with an attraction domain $\mathcal{U}$ defined by (14) and $T\left(x_{0}\right) \leq V\left(x_{0}\right) / q$ for $\quad x_{0} \in \mathcal{U}$, where $T(\cdot)$ is a settling-time function.

If a function $V$ is globally proper on $\Omega=\mathbb{R}^{n}$ then the inequality (15) implies global finite-time stability of the system (4).

Obviously, a settling-time function of finite-time stable system is a Lyapunov function in a generalized sense. Theorem 5 operates with a very large class Lyapunov functions. However, its conditions are still rather conservative. For example, the settling-time function from the next example does not satisfy Theorem 5. However, its simple discontinuous modification transforms the corresponding settling time function to a Lyapunov function in the sense of Theorem 5.

Example 3. Consider the so-called twisting system (Levant [1993])

$$
\left(\begin{array}{c}
\dot{x}_{1} \\
\dot{x}_{2}
\end{array}\right) \in F\left(x_{1}, x_{2}\right)=\left(\begin{array}{c}
y \\
-\left(2 \overline{\operatorname{sign}}\left[x_{1}\right] \dot{+} \overline{\operatorname{sign}}\left[x_{2}\right]\right)
\end{array}\right),
$$

which is finite-time stable with the settling-time function (Polyakov and Poznyak [2012]):

$T_{t w}\left(x_{1}, x_{2}\right)=p \sqrt{\left|x_{1}\right|+\frac{x_{2}^{2}}{2\left(2+\operatorname{sign}\left[x_{1} x_{2}\right]\right)}}+\frac{\left|x_{2}\right| \operatorname{sign}\left[x_{1} x_{2}\right]}{2+\operatorname{sign}\left[x_{1} x_{2}\right]}$,

where $p=4 \sqrt{2} /(3-\sqrt{3})$. The function $T_{t w}$ is globally proper, Lipschtz continuous outside the origin and continuously differentiable for $x_{1} x_{2} \neq 0$

$$
D_{F} T_{t w}=\frac{\partial T_{t w}}{\partial x_{1}} x_{2}-\frac{\partial T_{t w}}{\partial x_{2}}\left(2 \operatorname{sign}\left[x_{1}\right]+\operatorname{sign}\left[x_{2}\right]\right)=-1 \text {. }
$$

In the same time, $T_{t w}\left(x_{1}, x_{2}\right)$ does not satisfy (15), since $D_{F} T_{t w}\left(0, x_{2}\right) \bigcap \mathbb{R}_{+} \neq \emptyset$ for $x_{1}=0$. Applying generalized Clark's gradient (see, for example, Clarke [1990]) does not solves this problem.

However, if $p>\frac{4 \sqrt{2}}{3-\sqrt{3}}$ then the function $T_{t w}\left(x_{1}, x_{2}\right)$ is discontinuous on the line $x_{1}=0$ for $x_{2} \neq 0$, but it is globally proper and satisfies the conditions of Theorem 5 . Indeed, the equality (17) still holds and $D_{F} T_{t w}\left(0, x_{2}\right)=$ $\{-\infty\}$ for $x_{2} \neq 0, D_{F} T_{t w}\left(x_{1}, 0\right) \leq-1 / 3$ for $x_{1} \neq 0$. The Fig. 3 depicts a trajectory and the discontinuous Lyapunov function for twisting system. 


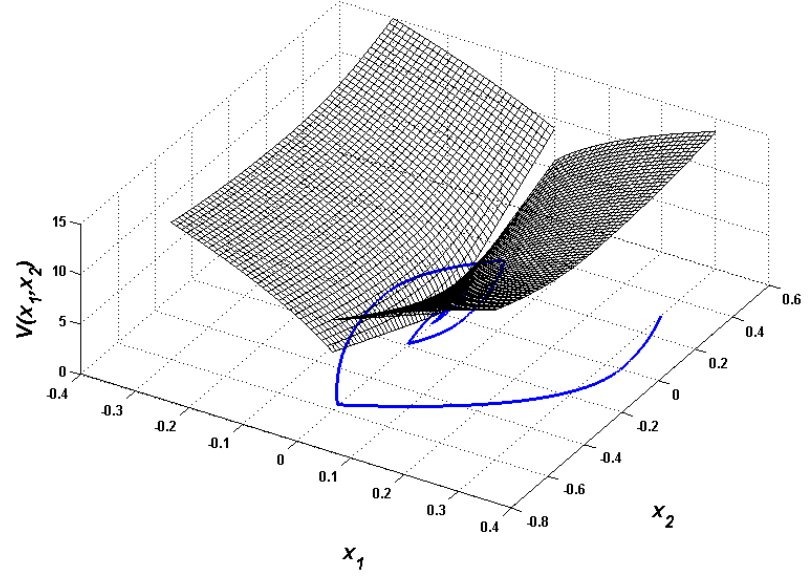

Fig. 1. A trajectory and the discontinuous Lyapunov function of twisting system

\section{CONCLUSIONS}

The paper presents the discontinuous Lyapunov function method for non-asymptotic stability analysis of differential inclusions. The example of discontinuous Lyapunov function is presented for twisting control system in order to demonstrate some advances of the method.

\section{REFERENCES}

M. A. Aizerman and E.S. Pyatnitskii. Foundations of theory of discontinuous systems i. Automation and Remote Control, 35:1066-1079, 1974.

A. Bacciotti and F. Ceragioli. Nonpathological Lyapunov functions and discontinuous caratheodory systems. Automatica, 42:453-458, 2006.

A. Bacciotti and L. Rosier. Lyapunov Functions and Stability in Control Theory. Springer, Berlin, 2005.

E. A. Barbashin. Lyapunov Functions. Moscow:Nauka [in Russian], 1970.

G. Bartolini and T. Zolezzi. Variable structure systems nonlinear in the control law. IEEE Transactions on Automatic Control, 30(7):681-684, 1985.

S.P. Bhat and D.S. Bernstein. Finite time stability of continuous autonomous systems. SIAM J. Control Optim., 38(3):751-766, 2000.

I. Boiko. Discontinuous control systems : frequencydomain analysis and design. Boston : Birkhauser, 2009.

V. Chellaboina, A. Leonessa, and W. Haddad. Generalized Lyapunov and invariant set theorems for nonlinear dynamical systems. Systems \& Control Letters, 38:289295, 1999.

S.B. Choi and J.K. Hedrick. Robust throttle control of automotive engines. ASME Journal of Dynamic Systems, Measurement, and Control, 118:92-98, 1996.

F. Clarke. Optimization and Nonsmooth Analysis. SIAM, Philadelphia, 1990.

F. Clarke, Y. S. Ledyaev, R. Stern, and P. Wolenski. Qualitative properties of trajectories of control systems: A survey. Journal of Dynamical and Control Systems, 1(1):1-48, 1995.

F. H. Clarke, Yu. S. Ledyaev, and R.J. Stern. Asymptotic stability and smooth Lyapunov functions. Journal of differential Equations, 149:69-114, 1998.
A. F. Filippov. Differential Equations with Discontinuous Righthand Sides. Kluwer Academic Publishers, 1988.

I. Flugge-Lotz. Discontinuous automatic control. Princeton University Press, 1953.

W. Hahn. Stability of Motion. Springer-Verlag Berlin Heidelberg, New York, 1967.

W.P.M.H. Heemels and S. Weiland. Input-to-state stability and interconnections of discontinuous dynamical systems. Automatica, 44:3079-3086, 2008.

A. Levant. Sliding order and sliding accuracy in sliding mode control. International Journal of Control, 58(6): 1247-1263, 1993.

A. M. Lyapunov. The general problem of the stability of motion. Taylor \& Francis, 1992.

B. S. Mordukhovich. Variational Analysis and Generalized Differentiation I: Basic Theory. Springer, 2006.

J. Moreno and M. Osorio. Strict Lyapunov functions for the super-twisting algorithm. IEEE Transactions on Automatic Control, 57:1035-1040, 2012.

E. Moulay and W. Perruquetti. Finite-time stability conditions for non-autonomous continuous systems. International Journal of Control, 81(5):797-803, 2008.

I.P. Natanson. Theory of functions of a real variable. Frederick Ungar Publishing Co., New York, 1955.

Y. I. Neimark. Note on A. Filippov paper. In Proceedings of 1st IFAC Congress II. Butterworths, London, 1961.

Y. Orlov. Finite time stability and robust control synthesis of uncertain switched systems. SIAM J. Control Optim., 43(4):1253-1271, 2005.

A. Polyakov and A. Poznyak. Lyapunov function design for finite-time convergence analysis: "twisting" controller for second order sliding mode realization. Automatica, 45:444-448, 2009a.

A. Polyakov and A. Poznyak. Reaching time estimation for "super-twisting" second order sliding mode controller via Lyapunov function designing. IEEE Transactions on Automatic Control, 54(8):1951-1955, 2009b.

A. Polyakov and A. Poznyak. Unified Lyapunov function for a finite-time stability analysis of relay second-order sliding mode control systems. IMA Journal of Mathematical Control and Information, 29(4):529-550, 2012.

A.S. Poznyak. Advanced Mathematical Tools for Automatic Control Engineers. Volume 1: Deterministic Technique. Elsevier, 2008.

E. Roxin. On finite stability in control systems. Rendiconti del Circolo Matematico di Palermo, 15:273-283, 1966.

D. Shevitz and B. Paden. Lyapunov stability theory of nonsmooth systems. IEEE Transactions on Automatic Control, 39(9):1910-1914, 1994.

Y. Z. Tsypkin. Relay Control Systems. Cambridge University Press, 1984.

V. Utkin, J. Guldner, and J. Shi. Sliding Mode Control in Electro-Mechanical Systems. CRC Press, 2009.

R. E. Vinograd. The inadequacy of the method of characteristic exponents for the study of nonlinear differential equations. Math. Sbornik, 41(83):431-438, 1957.

S.C. Zaremba. Sur une extension de la notion d'equation differentiel. Comptes Rendus Acad. Sc., 199:545-548, 1934.

Q. J. Zhu. Lower semicontinuous lyapunov functions and subdifferential calculus. In 41st LEEE Conference on Decision and Control, Las Vegas, Nevada USA, 2002. 